\title{
Polydisperse Spray Flames in Vortex Flows
}

\author{
Yuval Dagan ${ }^{1, *}$, David Katoshevski², J. Barry Greenberg ${ }^{3}$ \\ ${ }^{1}$ Department of Mechanical Engineering, Massachusetts Institute of Technology, \\ Cambridge, MA 02139, USA \\ ${ }^{2}$ Environmental Engineering Unit, Ben-Gurion University of the Negev, Beer-Sheva, Israel \\ ${ }^{3}$ Faculty of Aerospace Engineering, Technion - Israel Institute of Technology, Haifa, Israel \\ ${ }^{*}$ Corresponding author: yuvalda@mit.edu
}

\begin{abstract}
A new mathematical analysis of the dynamics of laminar spray diffusion flames in the vicinity of a vortex flow field is presented. The governing equations for a spray evaporating in an unsteady vortex are studied. New similarity solutions are found for the dynamics of the spray and the flame it supports. Analytical solutions for the spray flames are derived using Schvab-Zeldovich parameters, through which the radial evolution of the flames is found. The results based on the solution reveal the significant influence the droplets size has on the diffusion flame dynamics in the vicinity of vortical flows.
\end{abstract}

\section{Introduction}

The dynamics of evaporating sprays play an important role in many practical combustion applications such as gasturbines and swirl combustion chambers. The interactions between a gaseous diffusion flame and a vortex flow-field were extensively studied [1, 2, 3]. In a previous study of Dagan et al. [4], unsteady turbulent spray-flame instability in a concentric jet combustion chamber was studied using large eddy simulations (LES). In their study, droplet grouping and ligament structures were identified in the vicinity of vortices in large recirculation zones.

Using direct numerical simulations (DNS) of Diesel spray combustion in the vicinity of a recirculation zone, Shinjo et al. [5] showed that when droplets are larger than the Kolmogorov microscale, mixing is strongly enhanced by the presence of droplets and fuel vapor clusters are likely to form quickly when the droplet number density is high. They suggested that external group combustion is likely to occur near the recirculation zone. The effects of droplet clustering on evaporation were thoroughly discussed by Sirignano [6] and Harstad et al. [7]. Clusters of droplets are formed in vortical flows, as they accelerate towards the outer region of the vortex[8]. The dynamics of dropletvortex interactions and their influence on the structure of an evaporating spray was numerically studied by [9]. Droplet-vortex interaction in the Karman-vortex street was studied by Burger et al. [10], using DNS and a theoretical approach imposing a harmonically oscillating flow field. Recently, Franzelli et al. [11] numerically characterized the regimes of spray flame-vortex interactions. They used a two-dimensional Oseen type vortex in their study. However, their study relates to a vortex moving perpendicularly through an opposed flow spray sheet. These studies emphasize the need for a more fundamental understanding of the influence of droplet dynamics on combustion in vortical environments and recirculating flows, which appear in turbulent, as well as laminar environments.

The objective of the present work is to analytically study the influence of a vortex flow-field has on the evaporation and combustion of polydisperse sprays. A new mathematical formulation for the dynamics of polydisperse sprays in the vicinity of a vortex flow field is presented. The governing equations for a polydisperse spray evaporating in an unsteady vortex and the diffusion flame they support are studied and new similarity solutions are found for the dynamics of the spray flames, using the sectional approach (following Tambour, Greenberg and Katoshevski [14, $15,16,17])$. Finally, preliminary results are shown for the influence of polydispersity on the reacting diffusion sprayflames in the vicinity of vortical flows.

\section{Governing equations \\ Gas phase}

The equations for a polydisperse spray evaporating in a two-dimensional unsteady axisymmetric vortex flow are presented. A polar coordinate system $(r, \theta)$ is employed in the following derivation of equations. As a result of our assumption of an axisymmetric flow, all derivatives with respect to the angular coordinate $\theta$ are assumed zero.

A constant density for the host gas is assumed. The constant density assumption is frequently adopted in diffusion flame studies under conditions in which the fuel and the oxidant are heavily diluted so that the heat released by chemical reaction is small in comparison with the thermal energy of the mixture and gas expansion is negligible. Under these assumptions, the governing gas-phase equations are

Gas-phase momentum:

$$
\begin{aligned}
& \frac{\partial v_{r}}{\partial t}+v_{r} \frac{\partial v_{r}}{\partial r}-\frac{v_{\theta}^{2}}{r}=-\frac{1}{\rho} \frac{\partial p}{\partial r}+\nu \frac{\partial}{\partial r}\left(\frac{1}{r} \frac{\partial}{\partial r}\left(r v_{r}\right)\right)-\sum_{j=1}^{N_{s}} \tilde{Q}_{j} F_{r, j}-\dot{S}_{e v, r} \\
& \frac{\partial v_{\theta}}{\partial t}+v_{r} \frac{\partial v_{\theta}}{\partial r}+\frac{v_{r} v_{\theta}}{r}=\nu \frac{\partial}{\partial r}\left(\frac{1}{r} \frac{\partial}{\partial r}\left(r v_{\theta}\right)\right)-\sum_{j=1}^{N_{s}} \tilde{Q}_{j} F_{\theta, j}-\dot{S}_{e v, \theta}
\end{aligned}
$$


where $\rho$ is the gas-phase density, $v_{r}$ is the gas radial velocity, $v_{\theta}$ is the tangential velocity of the host gas, $\nu$ is the kinematic viscosity and $p$ is the pressure. $\tilde{Q}_{j}$ denotes mass fraction of the liquid fuel in size section $j$ and $N_{s}$ is the total number of sections. $\dot{S}_{e v}$ accounts for the momentum transferred to the host gas by the vapors. $F_{r, j}$ and $F_{\theta, j}$ describe the interaction between the gas phase and the droplets of size section $j$ in the radial and tangential directions, respectively, being proportional to the relative velocity between the droplets and the gas

$$
F_{r, j}=\tau_{j}^{-1}\left(v_{r}-u_{r, j}\right) ; \quad F_{\theta, j}=\tau_{j}^{-1}\left(v_{\theta}-u_{\theta, j}\right)
$$

where $u_{r, j}$ and $u_{\theta, j}$ are the radial and tangential velocities of the droplets of section $j$, respectively. $\tau_{j}$ is the sectional droplet relaxation time-scale.

The conservation equations for the vapour mass fraction of the fuel, $m_{f}$ and the oxidizer, $m_{o}$, and energy equation are given by

$$
\begin{aligned}
& \frac{\partial m_{f}}{\partial t}+\frac{1}{r} \frac{\partial}{\partial r}\left(r v_{r} m_{f}\right)=D_{f} \frac{1}{r} \frac{\partial}{\partial r}\left(r \frac{\partial m_{f}}{\partial r}\right)+C Q-\frac{1}{\rho} \tilde{S}_{R, f} \\
& \frac{\partial m_{o}}{\partial t}+\frac{1}{r} \frac{\partial}{\partial r}\left(r v_{r} m_{o}\right)=D_{o} \frac{1}{r} \frac{\partial}{\partial r}\left(r \frac{\partial m_{o}}{\partial r}\right)-\frac{1}{\rho} \tilde{S}_{R, o} \\
& \frac{\partial T}{\partial t}+\frac{1}{r} \frac{\partial}{\partial r}\left(r v_{r} T\right)=\frac{k}{\rho C_{p}} \frac{1}{r} \frac{\partial}{\partial r}\left(r \frac{\partial T}{\partial r}\right)-\frac{h_{v}}{C_{p}} C Q+\frac{1}{\rho C_{p}} \tilde{S}_{R, T}
\end{aligned}
$$

following Katoshevski et al. [16]. $T$ is the temperature, $k$ and $C_{p}$ are the gas conduction coefficient and specific heat at constant pressure, respectively. $h_{v}$ accounts for the latent heat of vaporization, $D_{f}$ and $D_{o}$ are the diffusion coefficients, and we assume all species have the same $C_{P}$ value. The $S_{R}$ terms are the reaction source terms for each equation, based on the assumption of a global reaction of the form Fuel $+\zeta_{s}$ Oxidant $\rightarrow$ Products, where $\zeta_{s}$ is the stoichiometric coefficient. However, as will be showed in the following sections, Schvab-Zeldovich type variables will be formulated in the similarity coordinate. Hence, an explicit treatment of the reaction terms will not be required.

\section{Liquid spray phase}

In the present study the equations for the reacting flow and their solutions are obtained only for a mono-sectional case. However, the sectional equations for the dynamics of sprays are presented here in a polydisperse formulation for the sake of generality. The multi-size droplet population is represented by a set of $N_{s}$ sectional conservation equations of the form

$$
\frac{\partial \tilde{Q}_{j}}{\partial t}+\frac{1}{r} \frac{\partial}{\partial r}\left(r u_{r, j} \tilde{Q}_{j}\right)=-C_{j} \tilde{Q}_{j}+B_{j, j+1} \tilde{Q}_{j+1} ; \quad j=1,2, \ldots, N_{s}
$$

where the coefficient $B_{j, j+l}$ accounts for droplets from section $(j+1)$ which are added to section $j$ during their evaporation, whereas $C_{j}$ accounts for evaporation of droplets within section $j$ and for droplets that move from section $j$ to section $(j-1)$.

In reality, the evaporation coefficient is a complicated function of the temperature differential between the droplets and the surrounding gas, the diffusivity and other properties of the fuel and its surroundings. Here the $d^{2}-$ law underlies the definition of the evaporation coefficient. Reasonably accurate estimates of droplet size and vaporization time do provide some evidence of the validity of this law even under transient temperature conditions [15, 21, 22]. In addition, Labowsky [19] showed that the $d^{2}-l a w$ provides a reasonable prediction of the actual vaporization history of an interacting droplet, especially in the initial period of combustion. Considerable progress in going beyond the limitations of Labowsky's model and its findings [19] can be found in the work of [20]. In principle, any more sophisticated model could be used as a basis for constructing the sectional evaporation coefficients. Inclusion of such details is likely to affect our results in a quantitative rather than a qualitative way [24]. In a similar manner, no separate energy conservation equation is solved for the spray as the current model assumes instantaneous thermal adjustment of the gas-liquid mixture to a common temperature; although this may not always be the case in transient situations [23]. The present analysis is therefore limited to fairly volatile fuels and small droplets for which the $d^{2}-$ law is valid.

Under these assumptions, the spray sectional momentum equations are

$$
\begin{aligned}
& \frac{\partial u_{r, j}}{\partial t}+u r, j \frac{\partial u_{r, j}}{\partial r}-\frac{u_{\theta, j}^{2}}{r}=F_{r, j}+\frac{1}{\tilde{Q}_{j}}\left(S_{j, r}^{L, M}-u_{r, j} S_{j}\right) \\
& \frac{\partial u_{\theta, j}}{\partial t}+u_{r, j} \frac{\partial u_{\theta, j}}{\partial r}+\frac{u_{r, j} u_{\theta, j}}{r}=F_{\theta, j}+\frac{1}{\tilde{Q}_{j}}\left(S_{j, \theta}^{L, M}-u_{\theta, j} S_{j}\right)
\end{aligned}
$$

The last term in the brackets on the RHS of equations 8 and 9 represents loss of linear momentum $\left(u_{j} S_{j}\right)$ due to evaporation of droplets in section $j$, and the linear momentum $\left(S_{j}^{L M}\right)$ added to section $j$ due to droplets from higher sections that are added to section $j$ during their evaporation (see [16]).

Here,

$$
S_{j}=-C_{j} \tilde{Q}_{j}+B_{j, j+1} \tilde{Q}_{j+1}
$$

and

$$
\begin{aligned}
& S_{j, r}^{L, M}=-C_{j} \tilde{Q}_{j} u_{r, j}+B_{j, j+1} \tilde{Q}_{j+1} u_{r, j+1} \\
& S_{j, \theta}^{L, M}=-C_{j} \tilde{Q}_{j} u_{\theta, j}+B_{j, j+1} \tilde{Q}_{j+1} u_{\theta, j+1}
\end{aligned}
$$




\section{Similarity transformation of the gas and spray equations}

We shall now use a similarity variable, $\eta=r^{2} / 4 \nu t$ and rewrite the host gas velocities as a function of $\eta$ :

$$
\bar{v}_{r}(\eta)=\frac{r v_{r}}{\nu} ; \quad \bar{v}_{\theta}(\eta)=\frac{r v_{\theta}}{\nu} .
$$

Next, the droplet velocities are defined as

$$
\bar{u}_{r, j}(\eta)=\frac{r u_{r, j}}{\nu} ; \quad \bar{u}_{\theta, j}(\eta)=\frac{r u_{\theta, j}}{\nu} .
$$

Substituting the above definitions into the droplet momentum equations one obtains

$$
\begin{aligned}
& \bar{u}_{r, j}^{\prime}-\frac{1}{2 \eta} \bar{u}_{r, j}^{\prime} \bar{u}_{r, j}+\frac{1}{4 \eta^{2}} \bar{u}_{r, j}^{2}+\frac{1}{4 \eta^{2}} \bar{u}_{\theta, j}^{2}=-\frac{1}{4 \eta^{2}} \frac{1}{\nu^{2}} r^{3} F_{r, j}-\frac{1}{\eta} \frac{1}{Q_{j}} \bar{B}_{j, j+1} Q_{j+1}\left(\bar{u}_{r, j+1}-\bar{u}_{r, j}\right) \\
& \bar{u}_{\theta, j}^{\prime}-\frac{1}{2 \eta} \bar{u}_{\theta, j}^{\prime} \bar{u}_{r, j}=-\frac{1}{4 \eta^{2}} \frac{1}{\nu^{2}} r^{3} F_{\theta, j}-\frac{1}{\eta} \frac{1}{Q_{j}} \bar{B}_{j, j+1} Q_{j+1}\left(\bar{u}_{\theta, j+1}-\bar{u}_{\theta, j}\right)
\end{aligned}
$$

where the primes denote differentiation with respect to $\eta$. The drag terms are written explicitly as

$$
\frac{1}{\nu^{2}} r^{3} F_{r, j}=A_{j}\left(\bar{v}_{r}-\bar{u}_{r, j}\right) ; \quad \frac{1}{\nu^{2}} r^{3} F_{\theta, j}=A_{j}\left(\bar{v}_{\theta}-\bar{u}_{\theta, j}\right)
$$

where $A_{j}=\tau^{-1} r^{2} / \nu$. The characteristic time of the droplets is taken as $\tau=\frac{\rho_{d} d^{2}}{18 \mu_{g}}$, where $d, \rho_{d}$ and $\mu_{g}$ are the averaged droplet diameter in section $j$, liquid fuel density and the host gas viscosity, respectively. It can be demonstrated that for the range of radii involved $A_{j}$ does not change much. In order to obtain the spray equations in terms of $\eta$ only, the sectional evaporation coefficients are defined as

$C_{j}=\bar{C}_{j} / t, \quad B_{j, j+1}=\bar{B}_{j, j+1} / t$. The use of an inverse time dependence of the sectional evaporation coefficients is reasonable in a general sense as evaporation will indeed peter out after sufficient droplet life time has elapsed. Thus, in similarity coordinates, the spray equations take the form

$$
\left(1-\frac{1}{2 \eta} \bar{u}_{r, j}\right) Q_{j}^{\prime}-\frac{1}{2 \eta} \bar{u}_{r, j}^{\prime} Q_{j}=-\frac{1}{\eta}\left(-\bar{C}_{j} Q_{j}+\bar{B}_{j, j+1} Q_{j+1}\right)
$$

\section{Shvab-Zeldovich formulation of diffusion spray-flames in a vortex}

In the mono-sectional framework, the following set of equations are derived in the similarity coordinate, $\eta$, for the fuel mass fraction, the oxidizer mass fraction and temperature, respectively:

$$
\begin{aligned}
& \left(1-\frac{1}{2 \eta} \bar{u}_{r}\right) Q^{\prime}-\frac{1}{2 \eta} \bar{u}_{r}^{\prime} Q=\frac{1}{\eta} C Q \\
& \frac{m_{f}^{\prime \prime}}{S c_{f}}+\left(1+\frac{1}{\eta S c_{f}}-\frac{\bar{v}_{r}}{2 \eta}\right) m_{f}^{\prime}-\frac{\bar{v}_{r}^{\prime}}{2 \eta} m_{f}=-\frac{1}{\eta} C Q+\zeta_{f} M_{f} \frac{1}{\eta} S_{R} \\
& \frac{m_{o}^{\prime \prime}}{S c_{o}}+\left(1+\frac{1}{\eta S c_{f}}-\frac{\bar{v}_{r}}{2 \eta}\right) m_{o}^{\prime}-\frac{\bar{v}_{r}^{\prime}}{2 \eta} m_{o}=\zeta_{o} M_{o} \frac{1}{\eta} S_{R} \\
& \frac{T^{\prime \prime}}{S c_{o}}+\left(1+\frac{1}{\eta P r}-\frac{\bar{v}_{r}}{2 \eta}\right) T^{\prime}-\frac{\bar{v}_{r}^{\prime}}{2 \eta} T=\frac{h_{v}}{C_{P} T_{C}} C Q-\sum_{i} \zeta_{i} M_{i} h_{i} \frac{1}{\eta} S_{R}
\end{aligned}
$$

Next, we assume a unity Lewis number, $S c_{f}=S c_{o}=\operatorname{Pr}=S c$ and use the following notation:

$$
\hat{\zeta}=\frac{\zeta_{o} M_{o}}{\zeta_{f} M_{f}} ; \quad \hat{h}=\frac{h_{v}}{C_{P} T_{C}} ; \quad \zeta^{*}=\frac{\zeta_{o} M_{o}}{-1 / \hat{h}} \sum_{i} \zeta_{i} M_{i} h_{i}+\zeta_{f} M_{f}
$$

Finally, defining two Schvab-Zeldovich variables

$$
\beta=\frac{1}{S c}\left(m_{f}-\frac{m_{o}}{\hat{\zeta}}\right), \quad \gamma=\frac{T}{\hat{h}}+m_{f}-\frac{m_{o}}{\zeta^{*}}
$$

one obtains the following equations

$$
\begin{aligned}
& \beta^{\prime \prime}+S c\left(1+\frac{1}{\eta S c}-\frac{\bar{v}_{r}}{2 \eta}\right) \beta^{\prime}-\frac{\bar{v}_{r}^{\prime}}{2 \eta} \beta=\frac{1}{\eta} C Q \\
& \gamma^{\prime \prime}+S c\left(1+\frac{1}{\eta S c}-\frac{\bar{v}_{r}}{2 \eta}\right) \gamma^{\prime}-\frac{\bar{v}_{r}^{\prime}}{2 \eta} \gamma=0
\end{aligned}
$$

The boundary conditions for the above functions are

$$
\begin{aligned}
& \beta(\infty)=\frac{1}{S c} m_{f, \infty} ; \quad \beta(0)=-\frac{1}{S c} \frac{m_{o, 0}}{\hat{\zeta}} \\
& \gamma(\infty)=\frac{T_{\infty}}{\hat{h}}+m_{f, \infty} ; \quad \gamma(0)=\frac{T_{f}}{\hat{h}}-\frac{m_{o, 0}}{\zeta^{*}}
\end{aligned}
$$




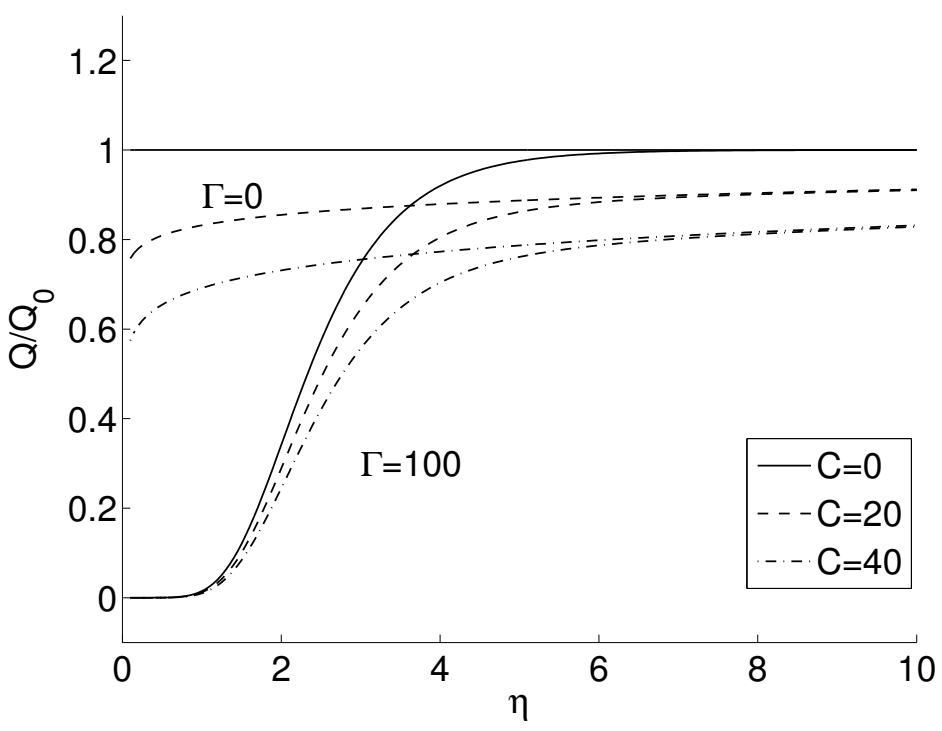

Figure 1. Liquid fuel mass fraction divided by the initial mass fraction $Q_{0}$ as a function of the similarity coordinate for different values of vortex intensity $\Gamma$ and evaporation coefficients $\bar{C}$.

It should be mentioned that for the actual solution we use the similarity variable boundaries from some small time and radius so that we avoid any singularity. The flame location is where $\beta=0$. This is the same as where the leading order solution (in terms of the usual Zeldovich number) locates the flame front.

In order to solve the equations for vortex spray dynamics analytically we shall first derive an expression for the droplet velocities $u_{r}$ and $u_{\theta}$ as follows. Appropriate manipulation of Eq. 15,16 leads to

$$
\bar{u}_{r}^{2} \bar{u}_{\theta}^{\prime}+\bar{u}_{\theta}^{2} \bar{u}_{\theta}^{\prime}=-A\left[\left(\bar{v}_{r}-\bar{u}_{r}\right) \bar{u}_{\theta}^{\prime}-\left(\bar{v}_{\theta}-\bar{u}_{\theta}\right) \bar{u}_{r}^{\prime}\right] .
$$

For the host velocity field, a two-dimensional Oseen-vortex will be used,

$$
\bar{v}_{\theta}=\bar{\Gamma}\left(1-e^{-\eta}\right) ; \quad \bar{v}_{r}=0 .
$$

which is also an exact solution of Eq. 2 for $\eta>>1$. Here, $\bar{\Gamma}=\Gamma / 2 \pi \nu$, where $\Gamma$ is the angular velocity. For the fine sprays under consideration, we assume that the droplets closely track the vortex flow-field in the transverse coordinate $\theta$. On the other hand, we expect the presence of the vortex to significantly increase the droplets radial velocity $u_{r}$, especially at lower values of $\eta$. Hence, droplets will not track the gas radial velocity which is initially assumed zero (Eq. 30). Thus, $\bar{u}_{\theta}=\bar{v}_{\theta}$ and equation 29 takes the following simple form

$$
\bar{u}_{r}^{2}+\bar{u}_{\theta}^{2}-A \bar{u}_{r}=0,
$$

from which $\bar{u}_{r}$ can be extracted.

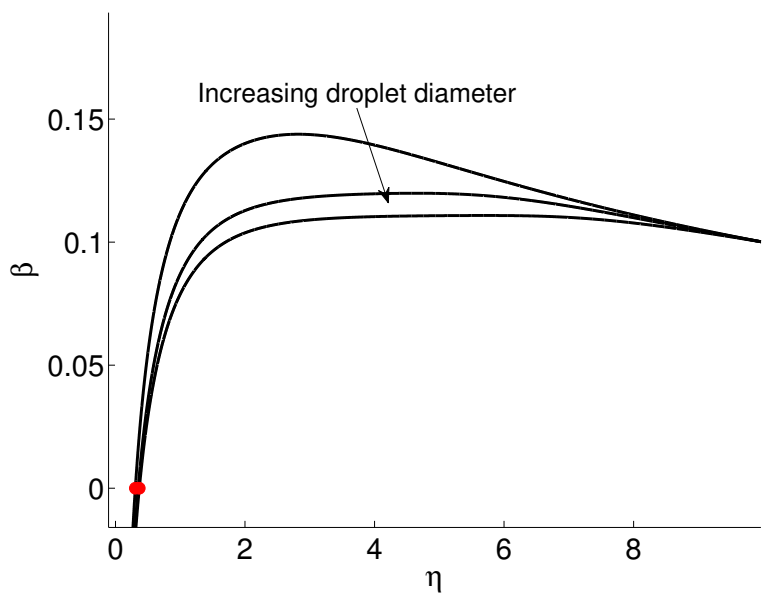

(a)

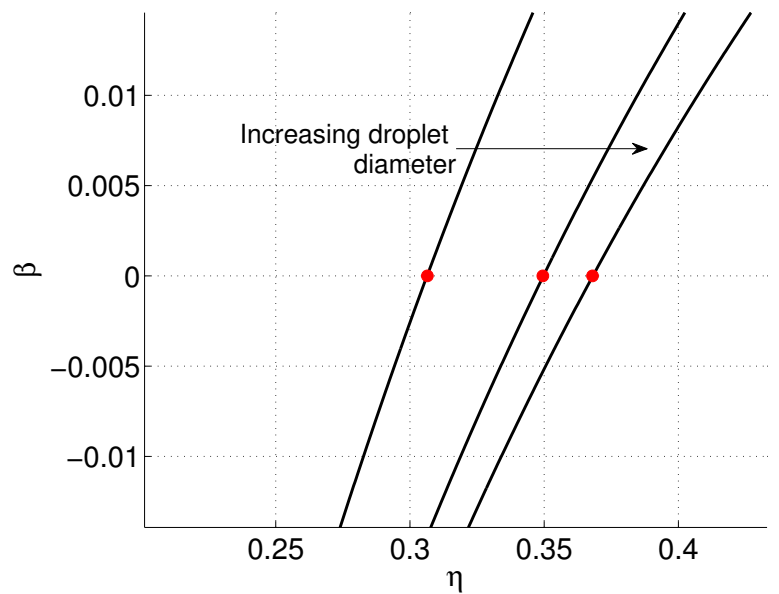

(b)

Figure 2. Schvab-Zeldovich function $\beta$ as a function of the similarity coordinate $\eta$ for three different initial droplet diameters: $d_{0}=10,50,100 \mu \mathrm{m}$. The flame is located where $\beta=0$ (shown in solid circles). (a) Whole region of the analysis (b) a closeup on the flame region at lower values of $\eta$. 


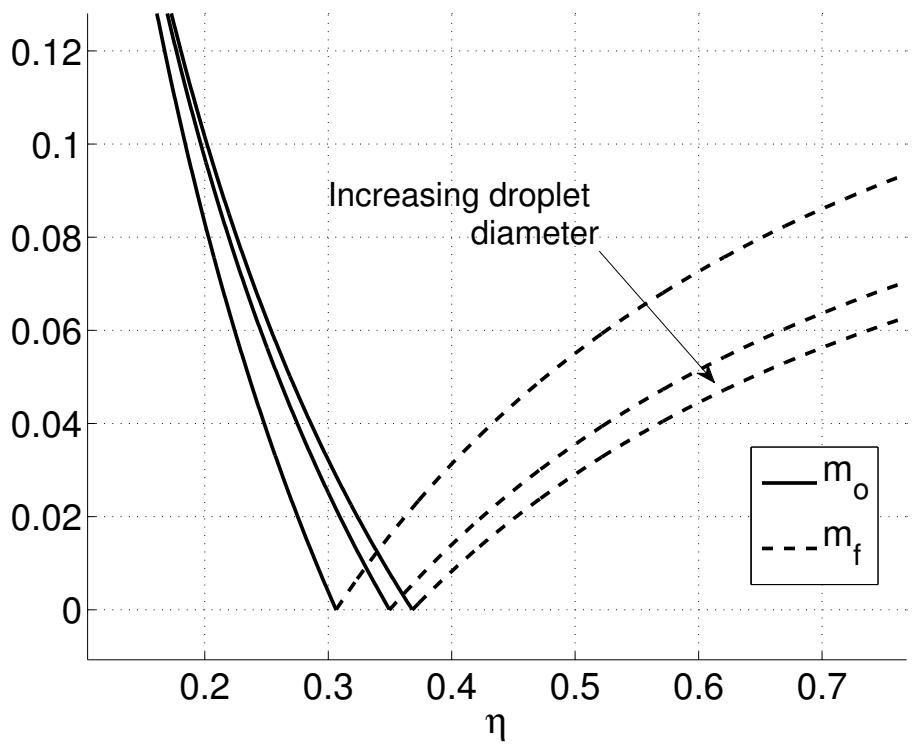

Figure 3. Profiles of fuel mass fraction $\left(m_{f}\right)$ and the oxidizer mass fraction $\left(m_{o}\right)$ in the similarity framework for three different initial droplet diameters: $d_{0}=10,50,100 \mu \mathrm{m}$.

Next, the equation for the liquid fuel mass fraction $Q$ (Eq. 19) is solved by separation of variables:

$$
Q^{\prime} / Q=\left(2 \bar{C}+\bar{u}_{r}^{\prime}\right) /\left(2 \eta-\bar{u}_{r}\right),
$$

from which $Q(\eta)$ can be solved by integration. Assuming $\bar{v}_{r}=\bar{v}_{r}^{\prime}=0$, we may also evaluate $\beta(\eta)$ numerically. The solution for the second Schvab-Zeldovich function, $\gamma$ is

$$
\gamma(\eta)=c_{3} E i(-S c \eta)+c_{4}
$$

where $E i$ is the exponential integral function, and $c_{1}, c_{2}, c_{3}$ and $c_{4}$ are integration constants found using the boundary conditions (Eq. 27, 28). In the present study equation 33 is also evaluated numerically.

\section{Results and discussion}

The liquid fuel mass fraction divided by the initial mass fraction $Q_{0}$ is presented in Figure. 1 as a function of the similarity coordinate for different values of vortex values of $\Gamma$ and evaporation coefficients $\bar{C}$. For non-evaporating droplets, $\bar{C}=0$, and zero vortex intensity, $\Gamma=0$, the liquid mass fraction does not change, as expected. On the other hand, for evaporating droplets and $\Gamma=0$ the liquid mass fraction decreases with decreasing values of $\eta$, which corresponds to increasing time values for a given radial location. The vortex intensity has a substantial effect, especially at lower values of $\eta$, in effect pushing the fuel droplets from the center outwards as time goes by. No droplets are found below $\eta \approx 1$. The calculation of liquid mass fraction was carried out between $0.1<\eta<100$. Only a a partial view is presented in the figure for clarity. Hence, for $\eta \rightarrow 100, Q / Q_{0} \rightarrow 1$.

\section{Polydispersity effect}

In our previous study [18] it was shown that in the vicinity of a vortical flow, spray dynamics is highly affected by the droplets' size, but also by the spray initial distribution, even when the same Sauter mean diameter is considered. It will therefore be interesting to capture the influence of initial droplet size on the propagation of the diffusion flame.

The equations for the dynamics of the polydisperse spray were derived here in the previous sections. In this section, as a first attempt to assess the influence polydispersity will have on the dynamics of the diffusion flame in the vicinity of vortical flows, the flame equations were solved analytically for different initial droplet sizes. It should be noted that the solution is essentially mono-sectional, and that the coupling effect between each section (e.g the intersection transfer of momentum) is out of the scope for this study and will not be considered here.

The Schvab-Zeldovich function $\beta$ is presented in Fig. 2a as a function of the similarity coordinate $\eta$ for three different initial droplet diameters: $d_{0}=10,50,100 \mu \mathrm{m}$. The droplet size effect on the flame location (at $\beta=0$ ) is significant, pushing the flame away from the vortex core (which s located at $\eta=0$ ). This is demonstrated in a closeup view in Fig. 2b. Although small droplets acceleration due to the presence of a vortex is higher than that of larger droplets, it can be shown by solving equation 15 that the maximum radial velocity reached is higher for the larger droplets. This affects the flame, as the vortex tends to push the larger droplet farther away and thus reducing the available fuel vapor required for the flame.

Knowing the functional behaviour of $\beta(\eta)$, and assuming that the species are totally consumed at the flame zone, one can easily extract the fuel vapour and oxidizer mass fraction as a function of $\eta$. This is shown in Fig. 3 for lower values of $\eta$, where the flame is located. Results for three different initial droplet diameters are presented, showing a significant droplet size effect; for a given value of $\eta$ (i.e at a given time and radial location in the physical space) lower fuel mass fraction is observed for larger droplets. The flame is located at the point where all the fuel and oxidizer are consumed $\left(m_{o}=m_{f}=0\right)$.

Finally, solutions of the temperature distribution in the similarity framework for the same vortex intensities and evaporation coefficients are presented in Fig. 4. For larger droplets that are pushed farther than smaller droplets, the temperature curve is pushed to higher values of $\eta$, which means that for a given time the flame will be pushed farther away from the vortex core. Increased heat 


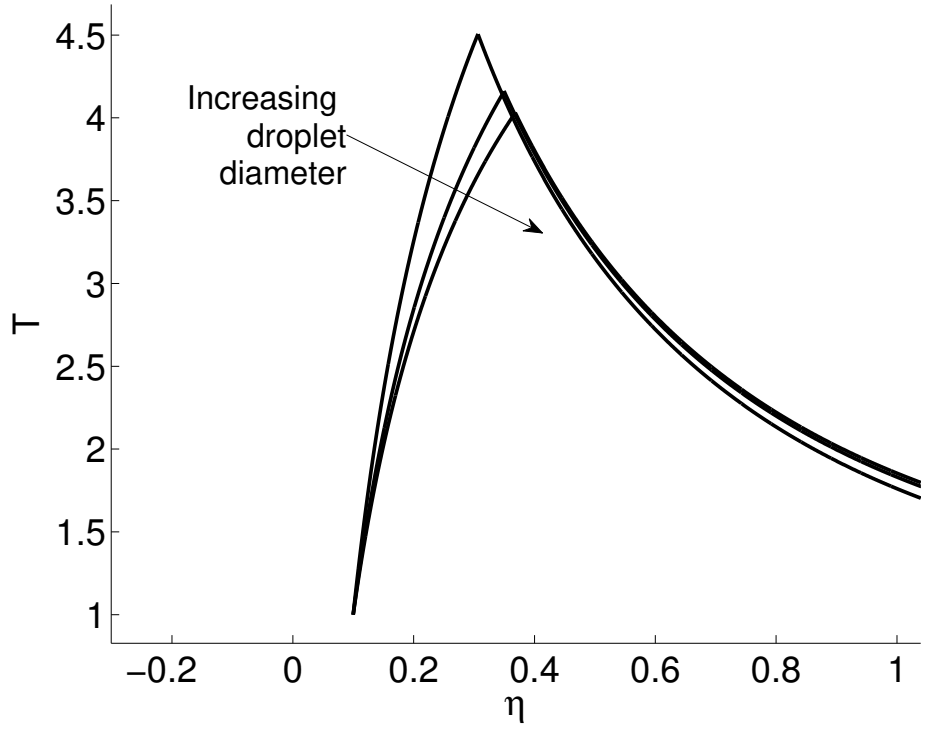

Figure 4. Solutions of the temperature distribution in the similarity framework for three different initial droplet diameters:

$$
d_{0}=10,50,100 \mu m \text {. }
$$

loss due to evaporation also plays an important role in lowering the temperature, which in some cases might lead to extinction. However, this is beyond the scope of the present study. Further work to elucidate the relative importance of the different factors controlling the spray-flame in vortex flows will be reported in the future.

\section{Conclusions}

A new mathematical analysis of the dynamics of laminar spray diffusion flames in the vicinity of a viscous vortex flow field was presented. The governing equations for sprays evaporating in an unsteady vortex were derived and new similarity solutions were found for the dynamics of the spray in a mono-sectional framework. New analytical solutions for the spray flames were obtained using Schvab-Zeldovich parameters, through which the radial evolution of the flames was found. The vortex flow field was shown to have a significant effect on the location and extent of the flame, pushing the droplets outwards and therefore shifting the flame location to larger radii for a given instant of time.

Spray flame with larger initial diameter droplets shows different flame dynamics; they are pushed away farther than spray flame with smaller droplets and exhibit lower temperatures. Certainly, future experimental data would be helpful in validating the theory presented in the current study.

\section{Nomenclature}

$A_{j} \quad$ dimensionless sectional drag coefficient

$B_{j, j+1}$ dimensionless sectional vaporization coefficient

$C_{j} \quad$ dimensionless sectional vaporization coefficient

$C_{P} \quad$ specific heat capacity at constant pressure $[\mathrm{J} / \mathrm{kgK}]$

$D$ diffusion coefficient $\left[\mathrm{m}^{2} / \mathrm{s}\right]$

$d \quad$ droplet diameter $[m]$

$F \quad$ function of $\eta$

$h \quad$ specific enthalpy $[\mathrm{J} / \mathrm{kg}]$

$k \quad$ thermal conductivity $[\mathrm{W} / \mathrm{mK}]$

$M \quad$ molecular weight $[\mathrm{g} / \mathrm{mol}]$

$m$ mass fraction

$N_{s} \quad$ number of sections

$\operatorname{Pr} \quad$ Prandtl number

$p \quad$ pressure $[\mathrm{Pa}]$

$Q_{j} \quad$ sectional liquid mass-fraction

$r$ radial coordinate $[m]$

$S \quad$ source term

$S c \quad$ Schmidt number

$T$ temperature $[K]$

$t \quad$ time $[s]$

$u \quad$ droplet velocity $[\mathrm{m} / \mathrm{s}]$

$v \quad$ host gas velocity $[\mathrm{m} / \mathrm{s}]$

\section{Greek symbols}

$\beta \quad$ Shvab-Zel'dovich function

$\Gamma \quad$ angular velocity $[\mathrm{rad} / \mathrm{s}]$ 


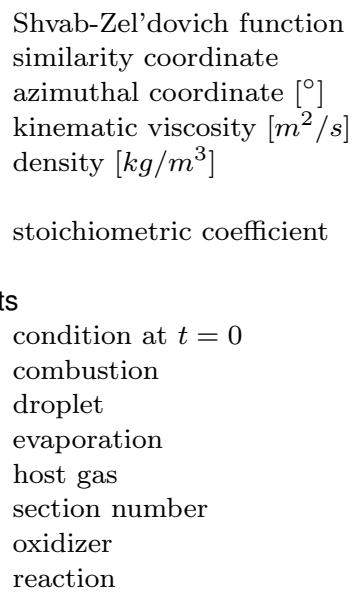

\section{References}

[1] Marble, F., 1985, Recent advances in the aerospace sciences, Springer, 395-413.

[2] Alain, M. and Candel, S., 1988, Combustion science and technology 60:79-96.

[3] Renard, P.H., Thevenin, D., Rolon, J.C. and Candel S., 2000, Progress in energy and combustion science 26: 225-282.

[4] Dagan, Y., Arad, E. and Tambour, Y., 2015, Proc. Combust. Inst. 35:1657-1665.

[5] Shinjo, J. and Umemura, A., 2013, Proc. Combust. Inst. 34:1553-1560.

[6] Sirignano, W.A., 1999, "Fluid Dynamics and Transport of Droplets and Sprays", Cambridge University press.

[7] Harstad, K. and Bellan, J., 2001, Combust. Flame 121:1861-1879.

[8] Bellan, J. and Harstad, K., 1991, Symposium (International) on Combustion 23:1375-1281.

[9] Park, T.W., Aggarwal, S.K. and Katta, V.R., 1996, Intl J. Heat Mass Transfer, 39:2205-2219.

[10] Burger, M., Schmehl, R., Koch, R., Wittig, S. and Bauer, H-J., 2006, Int. J. Heat Fluid FI. 27:181-191.

[11] Franzelli, B., Vié, A. and Ihme, M., 2014, Center for Turbulence Research Annual Research Briefs.

[12] Shiah, S. and Sichel, M., 1993, AIAA paper, 93-0901.

[13] Santoro, V., Kyritsis, D. and Gomez A., 2000, Proc. Combust. Inst. 28:1023-1030.

[14] Tambour, Y., 1985, Combust. Flame 60:15-28.

[15] Greenberg, J.B., Silverman, I. and Tambour, Y., 1993, Combust. Flame 93:90-96.

[16] Katoshevski, D. and Tambour, Y., 1993, Phys. Fluids, A 5:3085-3098.

[17] Greenberg, J.B., 2007, Combust. Flame, 148:187-197.

[18] Dagan, Y., Katoshevski, D. and Greenberg, J.B., 2016, ILASS - Europe, 27th Annual Conference on Liquid Atomization and Spray System, At Brighton, UK.

[19] Labowsky M., 1980, Combust. Sci. Technol., 22(5-6):217-26.

[20] Imaoka, R T and Sirignano, WA, 2005, Proc. Combust. Inst., 30(2):1981-1989.

[21] Law, CK, 1976, Combust. Flame, 26:17-22

[22] Law, CK,1977, Combust. Sci. Technol., 15(1-2):65-74.

[23] Abramzon, B and Sirignano, WA, 1989, 32(9):1605-1618.

[24] Greenberg, J. B. and Katoshevski, D., 2016, Combust. Theor. Model, 20(2):349-372. 